\title{
Making the change through acute medical team education: Inpatient risk assessment of stable pulmonary embolism cases at high risk of deterioration
}

\author{
Authors: Simon Millington, ${ }^{A}$ Andrew Lawson ${ }^{B}$ and Kumar Rajiv ${ }^{B}$
}

\section{Introduction}

The safety of optimal management of acute sub-massive or massive pulmonary embolism (PE) with thrombolytic agents remains unclear. ${ }^{1}$ Thrombolytic agents reduce mortality and improve right ventricular function, but increase the risk of major haemorrhage and stroke. ${ }^{2-4}$ Our project reviews current management of acute PE with evidence of myocardial necrosis and right ventricular strain, improving awareness by educating the medical teams and how best to investigate and treat such cases.

\section{Method}

A retrospective analysis of cases ICD-10 coded diagnoses of PE was carried out via data collected from diagnostic imaging reports, discharge summaries and case notes. Study cycles included the first cohort of 56 patients identified between August 2017 and July 2018, the second cohort of 91 patients between August 2018 and January 2019.

Educational and quality improvement interventions included presentation of data to acute and general internal medical teams and regional venous thromboembolism multidisciplinary teams, departmental teaching sessions, review and upgrade of trust policy on use of thrombolytic agents in sub-massive or massive PE. Data points of concern included evidence of myocardial necrosis via serum high-sensitivity troponin I (HS Trop I), transthoracic echocardiography (TTE) or computed tomography pulmonary angiography (CTPA) evidence of right ventricular strain, documented use of thrombolytic agents and any complications.

\section{Results}

Patients with large clot burden were identified in 26/56 (46\%) of cohort 1 cases and 55/91(60\%) of cohort 2 . Only $19(33 \%)$ cases in cohort 1 and $62(68 \%)$ in cohort 2 received HS Trop I investigation. TTE was completed in $9 / 56$ (16\%) of cases in cohort 1 and 17/91(18\%) in cohort 2 . These results indicate a poor initial utilisation of critical diagnostic investigations for risk stratifying acute PE. Following the quality improvement

Authors: ASheffield Teaching Hospitals NHS Foundation Trust, Sheffield, UK; ${ }^{B}$ Barnsley NHS Hospital Foundation Trust, Barnsley, UK intervention described, we observed significant improvement in the post-PE use of HS Trop I but not in TTE uptake.

Of particular interest were 20 patients in cohort 2 with a HS Troponin I of $>100 \mathrm{mmol} / \mathrm{L}$, indicating myocardial necrosis. Within these 20 patients were 11 mentions of right ventricular dysfunction on CTPA, and four on inpatient TTE. Overall 15 of these 20 patients met the criteria for thrombolysis.

Three acute PEs were thrombolysed with intravenous alteplase; two acute massive PEs and one sub-massive PE. There were no adverse outcomes and average length of stay was identical to that of patients not receiving thrombolysis.

\section{Discussion}

This real-world study highlights that optimal diagnostic investigations were previously grossly underutilised, potentially risking future morbidity and mortality. Clinical behaviours improve with education of the gold standards of risk stratification associated with this diagnosis. Our data identifies that thrombolysis seems to be reserved to shocked patients, with significant individual variance in using thrombolytic therapy, on a case-by-case basis, rather than on objective risk stratification. All cases where thrombolytic therapy was utilised proved to be safe and appropriate, with no subsequent morbidity or mortality.

\section{Conflicts of interest}

None declared.

\section{References}

1 Konstantinides SV, Meyer G, Becattini C et al. 2019 ESC Guidelines for the diagnosis and management of acute pulmonary embolism developed in collaboration with the European Respiratory Society (ERS). Eur Heart J 2019:ehz405 [Epub ahead of print].

2 Jaff MR, McMurtry MS, Archer SL et al. Management of massive and submassive pulmonary embolism, iliofemoral deep vein thrombosis, and chronic thromboembolic pulmonary hypertension: a scientific statement from the American Heart Association. Circulation 2011;123:1788-830.

3 Malik S, Bhardwaj A, Eisen M, Gandhi S. Advanced management options for massive and submassive pulmonary embolism. US Cardiology Review 2016;10:30-5.

4 Meyer G, Vicaut E, Danays T et al. Fibrinolysis for patients with intermediate-risk pulmonary embolism. $N$ Engl J Med 2014;370:1402-11. 